\title{
New Light On Numerical Boundary Conditions
}

\author{
K. Mazaheri* and P. L. Roe ${ }^{\dagger}$ \\ The University of Michigan \\ Ann Arbor, Michigan
}

$A 91-40773$

\begin{abstract}
We have made video films showing how the (computational) solution evolves to the steady state in a twodimensional airfoil problem. The video reveals very clearly that the path to convergence follows a simple, well-defined, repetitive pattern that begins almost immediately and is characterised by the passage of acoustic waves between the inner and outer boundaries. A perfect correlation is maintained between the timing of these waves, and a conventional residual history. We have conducted work aimed at preventing the regeneration of residuals by attenuating these continued reflections at the solid surface, by using a more general boundary condition which should coincide with the rigid wall $\mathrm{BC}$ in the steady limit. By analogy to the mass-less spring-damper system, it is shown that this acts as a soft wall boundary condition. There are two parameters which we optimize with the intention of minimizing reflected disturbances. The parameters are chosen based on a model problem of linear onedimensional acoustics, in the quarter-plane $t>0, x<0$. To check how these work at the discrete level, results of numerical experiments are presented, which agree with exact calculations quantitatively in the linear code, and qualitatively in the non-linear code. When applied to the calculation of two-dimensional lifting flows, we find CPU time can be reduced by more than $40 \%$.
\end{abstract}

\section{Introduction}

It is generally believed that one major factor delaying the convergence of CFD solutions to a steady state is the time taken for acoustic waves to travel repeatedly between the solid surface and the outer boundary. Fig 1 shows a familiar "oscillating path" toward convergence, that is plausibly explained by such behaviour. Much effort has therefore been devoted to the design of "absorbing" or "non-reflective" boundary conditions

\footnotetext{
* Graduate Research Assistant, Aerospace Engineering

IProfessor, Aerospace Engineering
}

to be applied at the outer boundary. These are, however, almost always based on some intuitive assumption about the manner in which the waves travel back and forth. For example the assumption is made in [1] that the waves travel normal to the boundary, and that a one-dimensional analysis is adequate. In $[2,3]$ acoustic waves originating at the solid surface are considered, so it is assumed that the angle at which the waves cross the boundary is known.

Although we agree that the removal of acoustic waves is a very important part of the convergence proccess, we are doubtful of the geometrical assumptions made. We believe that the first of these assumptions is almost never true (see section 2.2, Fig 8 ). The second may be true if a time-accurate path is followed, but makes no allowance for any form of preconditioning, such as local time-stepping.

In section 2.1 we will present a study in which residuals were visualized to reveal their true behaviour. Section 2.2 shows that important aspects of that behaviour can be predicted by a simple front-tracking calculation. Then in section 3 we present a new "soft wall" boundary procedure, to be applied at the inner boundary of the domain, with the object of minimizing wave reflections from a solid wall. The new boundary condition is applied to a subsonic two-dimensional airfoil problem in section 4.

\section{Experimental Observations}

\subsection{Residual Waves}

To discover the true state of affairs we have made video films showing how the solution evolves. Most of our results are for a simple first-order upwind scheme using local timestepping on a rather coarse grid (see Fig 18 ), because this code behaves rather simply and predictably, but we have made enough tests with other schemes and finer grids to feel some confidence in our general conclusions. Stills from one such video are 


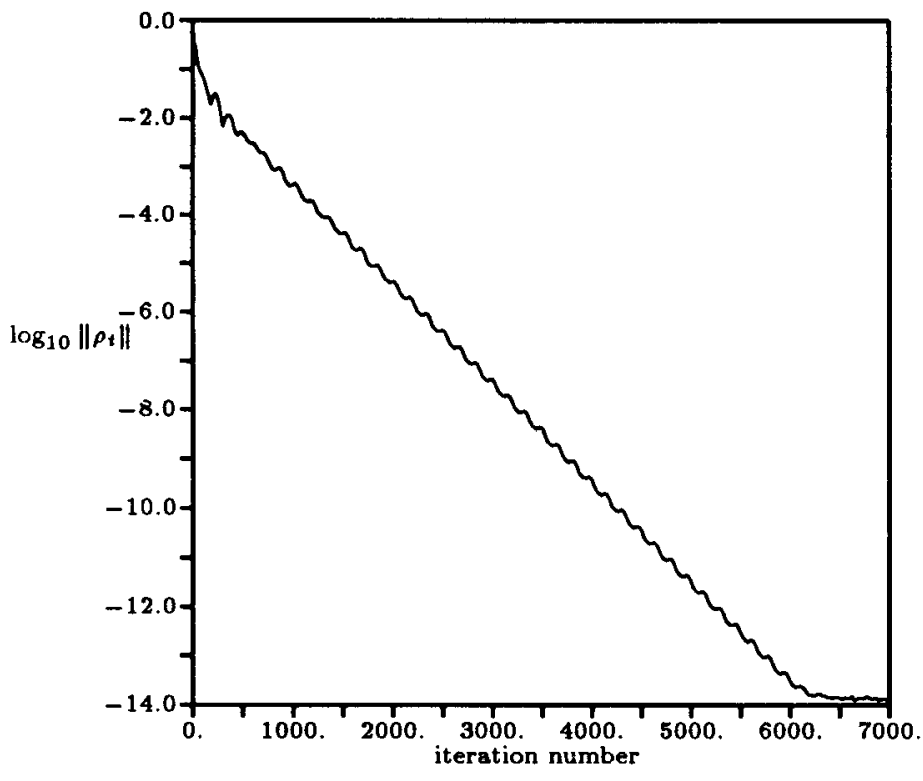

Figure 1: Residual history

shown in Figs 2, 9, 4, 5. The video reveals very clearly that the path to convergence follows a simple, welldefined, repetitive pattern that begins almost immediately. The video shows contours of Res $=$ Cell Area $\times\left|\rho_{t}\right|$, ranging between $R e s_{\max }$ and $R e s_{\max } / 300$ where $R e s_{\max }$ is the largest residual recently observed. The area weighting is used as a way of emphasizing the far field behaviour.

Given an initial solution equal everywhere to the free stream (including the circulation due to a vortex), acoustic disturbances can be seen to propagate both upstream and downstream. The waves going downstream are mainly expansive, whilst those going upstream are compressive waves focusing into a shockwave. Because of local time-stepping, this shock moves faster as it moves out into the coarser mesh, and becomes almost planar. This is what would be predicted by applying Huyghen's principle and allowing for local propagation speeds.

The average residual remains almost constant while the forward shock is moving outward; as the shock reaches the boundary, the residual falls rapidly. However, shortly afterward, a fresh set of waves originates (apparently spontaneously, but almost certainly triggered by reflected waves returning from the boundary) and the cycle repeats, although at a generally reduced amplitude. For the first six cycles, occupying the first 1000 iterations, there are minor variations, but after this each cycle repeats the previous one very faithfully. After the first cycle, almost all the largest residuals ap-

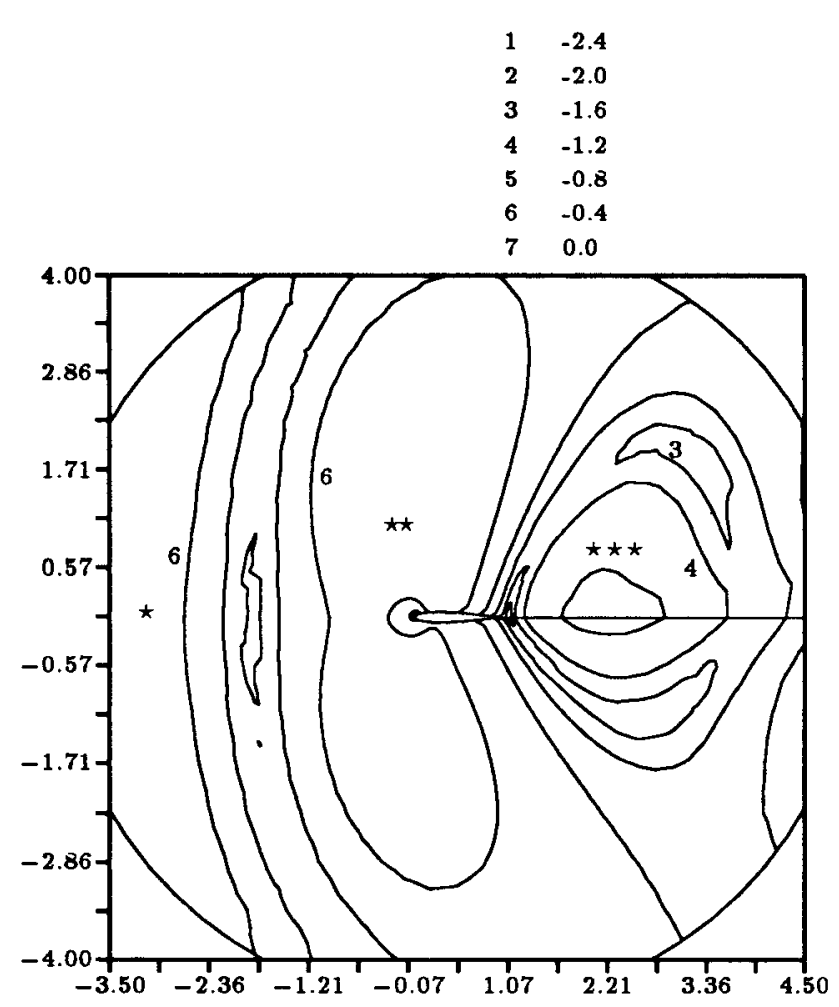

Figure 2: The residual contours, for 2D flow around NACA0012, $M=0.63, \alpha=2$ iteration No. $=1235$ using first-order Roe's upwind-scheme. Note that the contours are in logarithmic scale

pear in the front half of the flow. A perfect correlation is maintained between the timing of these waves, and the "beating" in a conventional residual history (as in Fig 1).

Fig 2 shows the residual pattern after 1235 explicit time steps. There regions labelled $(\star),(\star \star)$, and $(\star \star \star)$, will be tracked in time to gain insight into the convergence process. In $F i g$, they appear respectively ahead of the leftmost contour labelled " 6 ", within the second contour labelled " 6 ", and within the contour labelled " 4 ", and are the ones where the largest residuals are found. These regions of large residuals appear to be generated close to or somewhat behind the trailing edge, and then progress forward. In $F i g 2$, the region $(\star)$ is leaving the computational domain.

Fig 9 shows the residual pattern at time-step 1290. Region $(\star)$ has exited the domain, region $(\star \star)$ has moved upstream of the airfoil, and region $(\star \star \star)$ has grown substantially. By 1345 time-steps (as seen in Fig 4), region ( $\star \star)$ is exiting the outer boundary, upstream of the airfoil, and region $(\star \star \star)$ has grown, both in extent and in intensity. By 1410 time-steps (Fig 5), the pattern of Fig 2 has been restablished, with region $(\star \star)$ taking the place of the original region $(\star)$, and region 


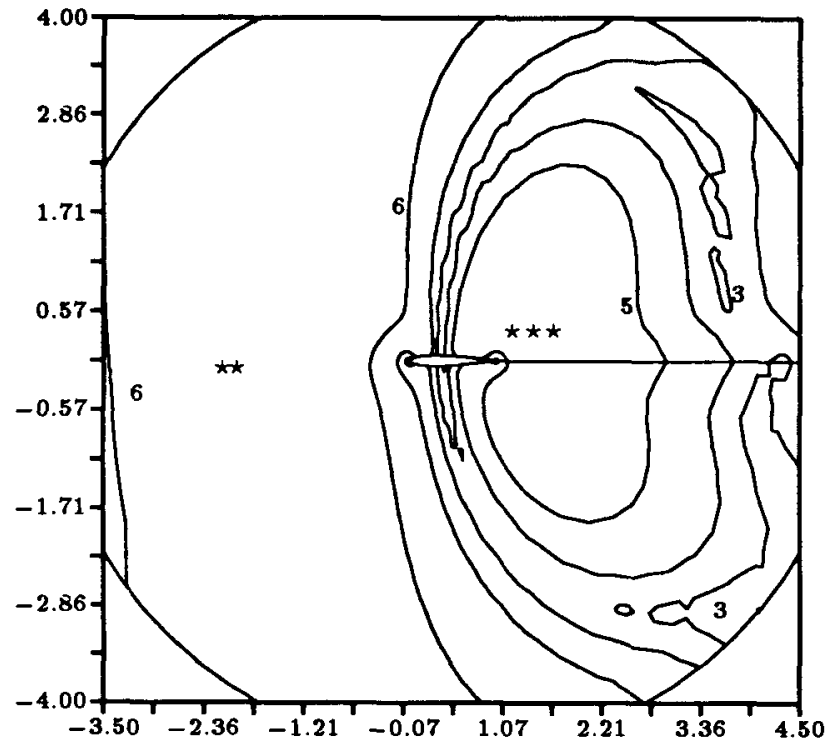

Figure 3: The residual contours, iteration No. $=1290$

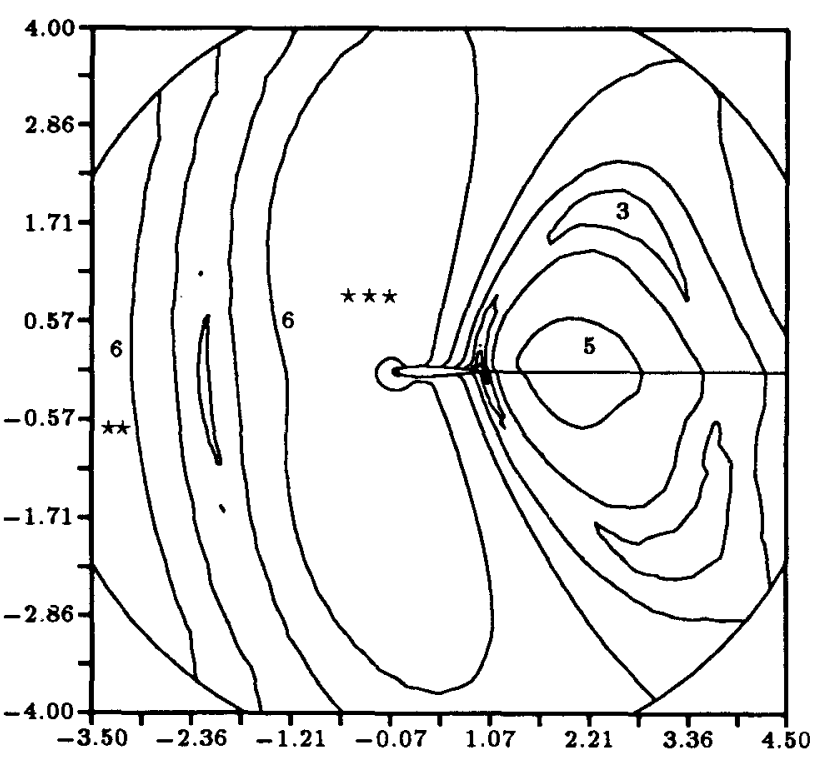

Figure 5: The residual contours, iteration No. $=1410$

$(\star \star \star)$ taking the place of the original region $(\star \star)$. The general level of residuals is of course lower, but because the plots are rescaled every 50 iterations, this is not reflected in the contours. The same cycle of events will be repeated until convergence is achieved.

If the calculation is made in single precision, then after about 2000 iterations, the residuals are close to machine zero, and the residual pattern seen in the video degenerates into random noise. However, if the calculation is performed in double-precision arithmetic (as in $F i g$ 1), then the pattern continues, and becomes increasingly regular.

The explanation of this particular sequence is a little mysterious. We conjecture that the seemingly spontaneous emission of waves from near the trailing edge is due to focussing of reflected waves that are themselves too weak to show up in the residual pattern (as displayed). To confirm this, we made calculations in which the shape of the wave front was explicitly tracked.

\subsection{Wave Front Tracking}

It proved difficult to visualize the path taken by the returning waves, because these are weaker than the outgoing waves. Instead, we used a technique that provides information directly about wave path. We solved numerically the eikonal equation

$$
\phi_{t}+[\mathbf{u} \cdot \nabla \phi+a|\nabla \phi|]=0
$$




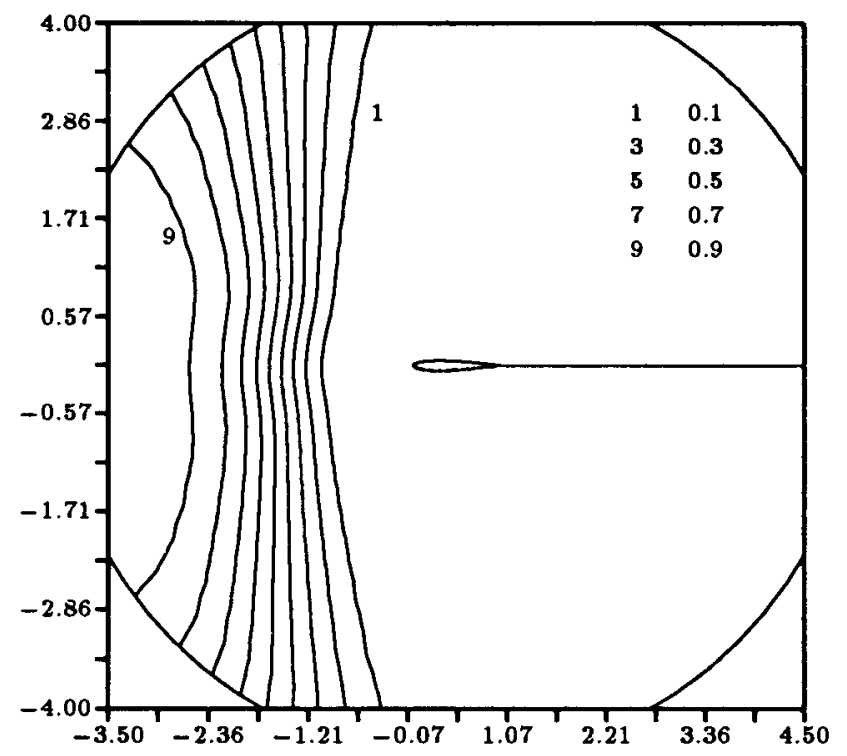

Figure 6: Contours of $\phi_{1}$ at iteration number 120

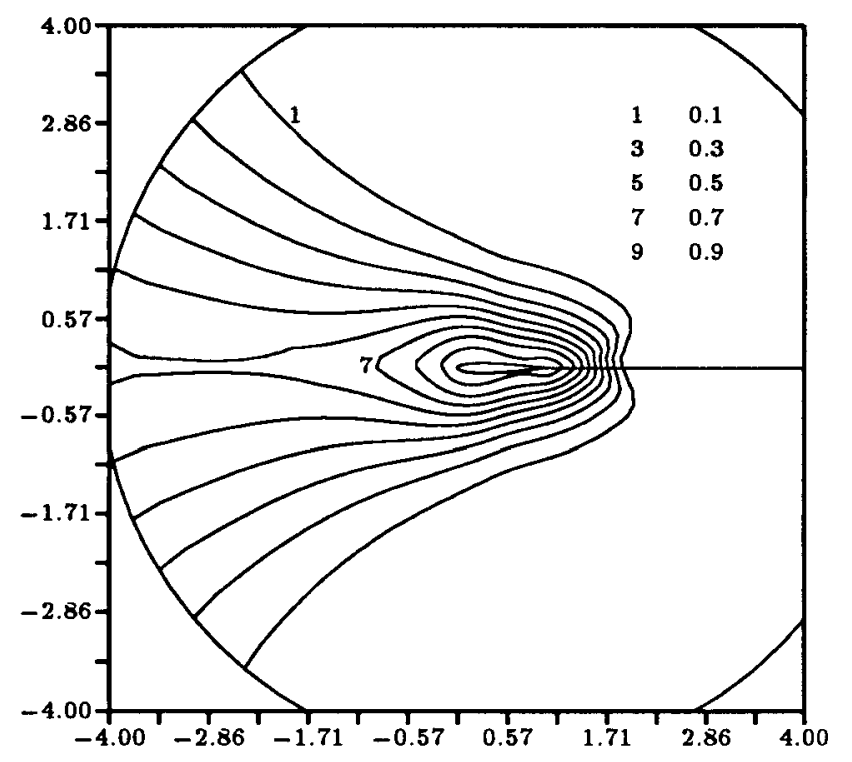

Figure 7: Contours of $\phi_{2}$ at iteration number 164
The level lines of $\phi$ follow acoustic wavefronts in a medium moving with velocity $\mathrm{u}(x, y)$ and having a soundspeed $a(x, y)$. We can also solve equation (1) using local timesteps, to simulate wave behaviour under local timestepping and using the values of $\mathbf{u}$ and $a$ from the Euler solver. The simulated outgoing waves agreed well with those seen in our Euler code. We used a device due to Harabetian [4] which allows reflected waves to be seen; he introduces two functions $\phi_{1}$ (for outgoing waves), and $\phi_{2}$ (for incoming waves) both satisfying equation (1), and sets

$$
\phi_{2}=\phi_{1}
$$

on the outer boundary. Throughout the flow at $t=0$ we take $\phi_{1}=1$ and $\phi_{2}=1$, but set $\phi_{1}=0$ on the airfoil surface. This generates a wavefront which propagates outward, and after hitting the outer boundary is reflected back toward the surface of the airfoil.

For the same test case as in section 2.1, Fig 6 shows the outgoing waves $\left(\phi_{1}\right)$ at a time that roughly corresponds to the Euler solution in Fig 3; the waves are found inside the region $(\star \star)$. It shows the same pattern of a forward-moving shock. The waves that initially travelled downstream have already left the domain, creating reflected waves $\left(\phi_{2}\right)$ that move rapidly around the coarse mesh near the outer boundary, and not quite so quickly along the line $y=0$. The effect is that the returning waves envelop the airfoil in the sort of pattern that can be seen in Fig 7 . Because of the fine mesh that usually surrounds any solid boundary, there is a tendency for the waves that arrive earliest to slow down and wait for their colleagues to catch up. This is the same mechanism by which waves arrive parallel to a beach. The returning waves therefore impact almost simultaneously on the airfoil. The moment at which this happens agrees closely with that at which the residuals in the Euler calculation are halted in their decline (i.e. the end of a "bump" in the conventional residual history in Fig 1). At this moment, also, a fresh set of outgoing waves will be generated to renew the cycle.

This wave-focussing is clearly the mechanism by which the residual history is tuned to a dominant frequency. There is satisfactory agreement between the period predicted from the eikonal computation, and that observed in the Euler code, both of about $170 \pm 5$ iterations (see Figs 1 and 7 ). However the history of the residual at a point has really a period twice that seen in Fig 1, or what is predicted by the eikonal calculations for one round trip by an acoustic wave. This is because compression waves reaching the outer boundary seem to be reflected as rarefaction waves, and vice versa, by our particular boundary procedure. It should also be noted that the present version of the eikonal equation computation fails to predict secondary waves that arise by interaction of primary waves with each other. Therefore some fine details are probably still missing from this account.

We also used the solution of the eikonal equation to predict the angles at which outgoing waves intersect the outer boundary. Fig 8 shows the angle between the wave normal $(\nabla \phi)$ and the boundary curve, at the 


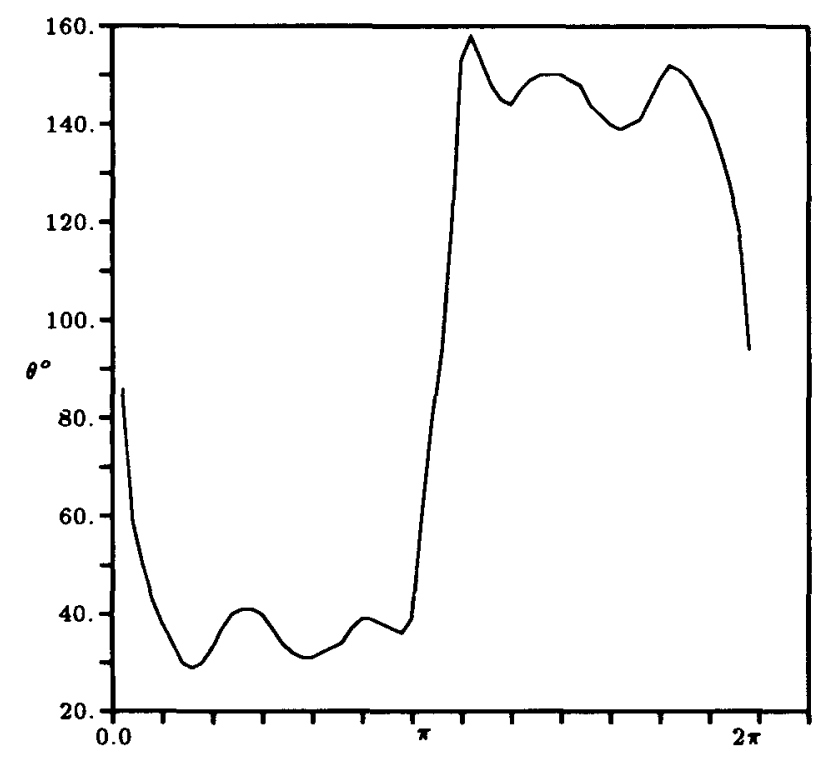

Figure 8: The incidence angle (relative to the grid angle) at different locations on the outer boundary

moment of refiection, as a function of the location on the outer boundary. From this figure it can be seen that it is a very poor approximation to take this angle as $90^{\circ}$.

\subsection{Residual Correlation}

All of these observations are convincing evidence that acoustic waves are responsible for an important part of the convergence process. An additional observation, suggesting that acoustic behaviour is almost completely dominant, comes from correlating the pressure and density residuals. For any purely acoustic disturbance, we should find that

$$
p_{t}=a^{2} \rho_{t}
$$

In fact, we find that this equation holds to within $1 \%$ on average over the flow field.

\section{Soft Boundary Conditions}

After one basic cycle, we can expect the residual to be reduced by a factor of something like

$$
R=R_{f} R_{o} R_{i}
$$

where $R_{f}$ is the attenuation due to numerical dissipation (whether by accident or design) as the waves traverse the flow twice, $R_{o}$ is the attenuation when the wave reflects from the outer boundary, and $R_{i}$ is the

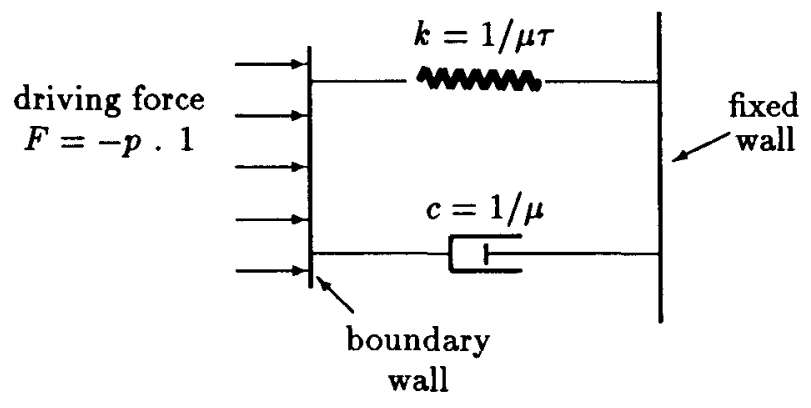

Figure 9: The mass-less spring-damper system

attenuation when the wave reflects from the inner solid boundary. Theoretically, it should be possible to make $R_{o}$ very small, but the difficulty of converting analytical insights into numerical procedure is notorious. For a recent innovation in this area, see the paper by Karni [5]. $R_{f}$ is presumably reduced by such techniques as multigrid, residual smoothing, and enthalpy damping.

At first sight, a solid inner boundary implies $R_{i}=1$, and we are not aware of any previous attempt to change this. However, we decided to experiment with replacing the boundary condition

$$
u_{n}=0 \text {. }
$$

by the more general boundary condition

$$
\frac{\partial u_{n}}{\partial t}+\frac{u_{n}}{\tau}=\frac{\mu}{\rho a} \frac{\partial p}{\partial t}
$$

which should coincide with equation 5 in the steady limit. $\tau$ and $\mu$ are parameters which we choose with the intention of minimizing reflected disturbances. It can be shown that the new boundary condition (equation 6 ) acts like a mass-less spring-damper system ( $F i g$ ).

\subsection{One Dimensional Analysis}

To choose $\tau$ and $\mu$, we consider the model problem of one-dimensional acoustics, governed by

$$
\begin{aligned}
u_{t}+\rho_{0}^{-1} p_{x} & =0 \\
p_{t}+\rho_{0} a_{0}^{2} u_{x} & =0
\end{aligned}
$$

in the quarter-plane $t>0, x<0$. We nondimensionalize everything by $\rho_{0}, a_{0}$ and a length scale, $l$ equal to distance between left and right boundaries. At $t=0$, we specify initial conditions $u=0, p=p_{0}+\bar{p} \sin (\omega x)$, and take equation ( 6 ) to be the boundary condition on $x=0$. The solution ( Fig 10) takes the form of the incident waves plus a set of reflected waves in the region 


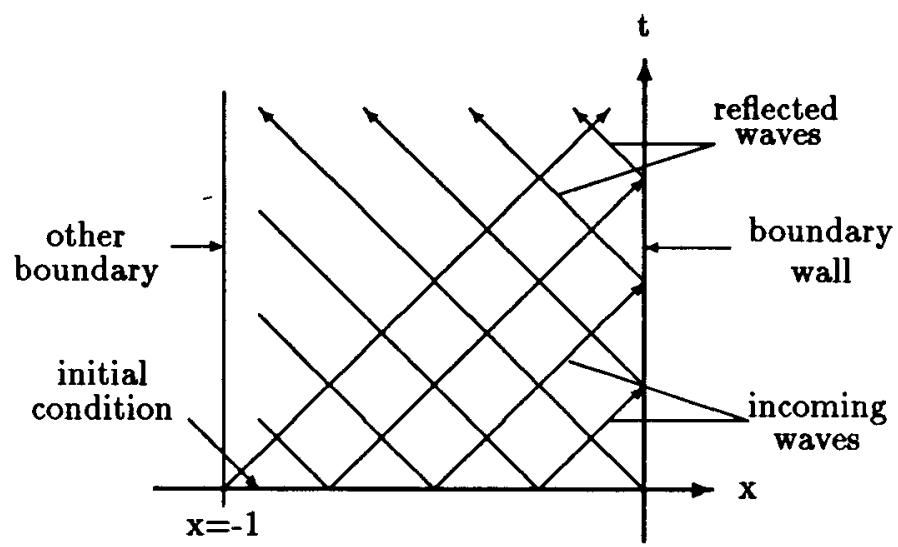

Figure 10: The sketch of the model

$x+a_{0} t>0$.

Both pressure and velocity on the wall vary sinusoidally with the same frequency $\omega$. Introducing the strength of returning waves $s_{r}(0, t)=\left(p(0, t)-p_{0}\right)-$ $u(0, t)$ and the strength of incoming waves as $s_{i}=\bar{p}$, we use $S=s_{r} / s_{i}$ as a measure of ratio of amplitude of the reflected waves versus the incident waves. Then, the amplitude of these waves is given by

$$
|S|=\left[\left(\omega^{\prime} \beta^{\prime}-1\right)^{2}+\beta^{\prime 2}\right]^{1 / 2}
$$

where $\omega^{\prime}=\omega \tau(1+\mu)$, and $\beta^{\prime}=\frac{2 \mu \omega \tau}{1+\omega^{2} \tau^{2}(1+\mu)^{2}}$. It shows that $|S|$ depends only on the parameters $\mu$ and $\omega \tau$. We see (Fig 11) that the amplitude is minimized (for any $\omega$ ) by taking $\mu=1.0$ and $\tau$ large compared to $\omega$. Note that $u$ is then proportional to the strength of the outgoing acoustic waves. Contours of $|S|$ are plotted in Fig 11 .

To check that this result was not confined to the special case of sinusoidal incident waves, we repeated the analysis for a Gaussian incident wave

$$
p(x, 0)=p_{0}+\bar{p} e^{-\beta(x+1 / 2)^{2}}
$$

Then the magnitude of the reflected wave at $x=0$ as a function of time is

$$
\begin{array}{r}
S(t)=e^{-\beta(-t+1 / 2)^{2}} \\
-2 \beta \mu^{\prime} e^{-t / \tau^{\prime}} \int_{0}^{t}\left(\frac{1}{2}-\zeta\right) e^{-\beta(-\zeta+1 / 2)^{2}+\zeta / \tau^{\prime}} d \zeta \\
=F\left(\mu^{\prime}, \beta, \tau^{\prime} ; t\right)
\end{array}
$$

where $\tau^{\prime}=\tau(1+\mu)$, and $\mu^{\prime}=\frac{2 \mu}{1+\mu}$. This is plotted in Fig 12. Again the preferred combination is $\mu=1$, and $\tau$ large. However, if $\tau$ is made too large, then after the impact of the wave is past, the wall velocity returns only slowly to zero. In fact, the parameter $r$ is loosely bounded by two considerations. One of these is that $\tau$ must not be much less than a typical timestep,

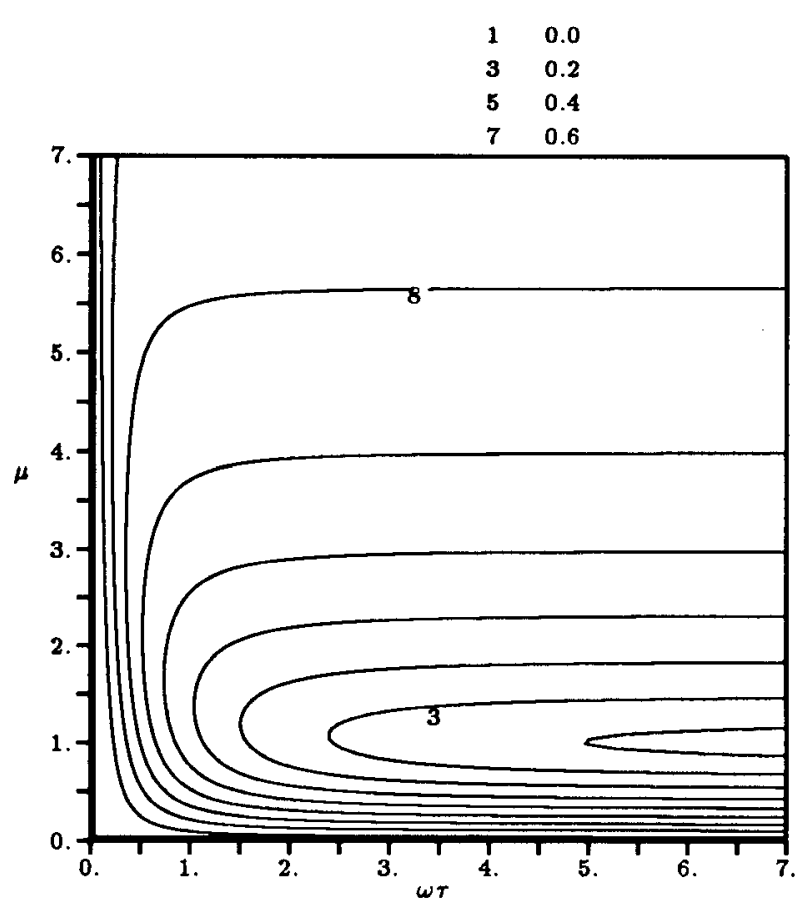

Figure 11: The strength of reflected waves for a sinusoidal initial condition for pressure distribution

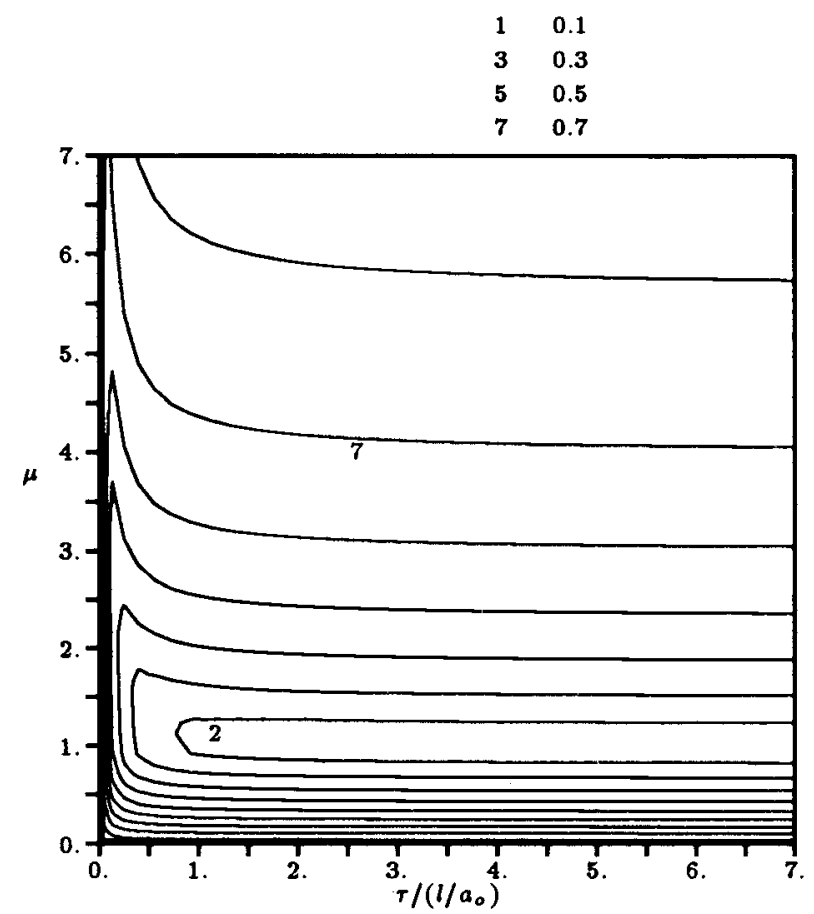

Figure 12: The strength of reflected waves for a Gaussian initial condition for pressure distribution 


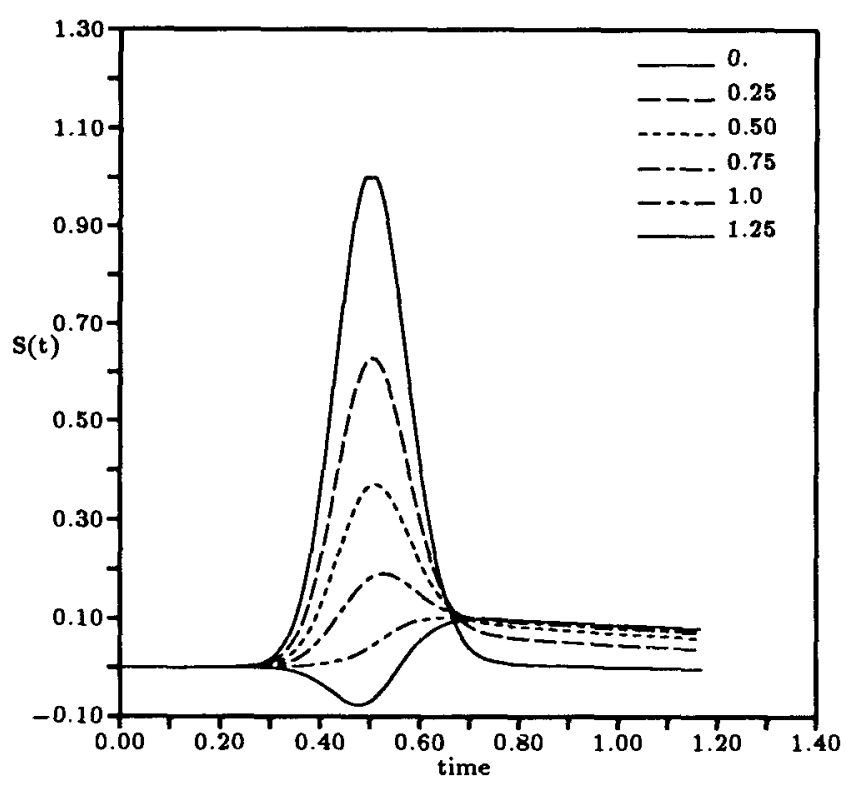

Figure 13: The strength of reflected waves for different values of $\mu$. Here $\tau / \Delta t=100$

otherwise we may have difficulty in modelling the equation. On the other hand $\tau$ should not be greater than the round-trip time for a wave to cross the domain, for then the next returning wave will not encounter the proper boundary.

Also, when we come to apply these boundary conditions to a two-dimensional example, we realize that the above analysis, although correct, is not the most helpful. For an input wave of essentially finite duration, not only the peak amplitude of the returning wave is significant, but also the amplitude at the moment when the incident wave ceases, and the returning wave begins to decay freely. This determines how quickly the wall returns to its unperturbed position. The onedimensional analysis (see $F i g$ 13) seemed to indicate a value of $\mu$ roughly equal to 0.5 providing a resonable compromise between these requirements, and this was also about the value that gave best convergence in our two-dimensional experiments. With more cells in the mesh, local timestepping needs more iterations to send the wave on a round trip, so that we can tolerate a slower return to rigidity. We have found that on meshes with greater numbers of mesh points, the optimal value of $\mu$ is greater than 0.5 , but the precise value does not seem to be very critical.

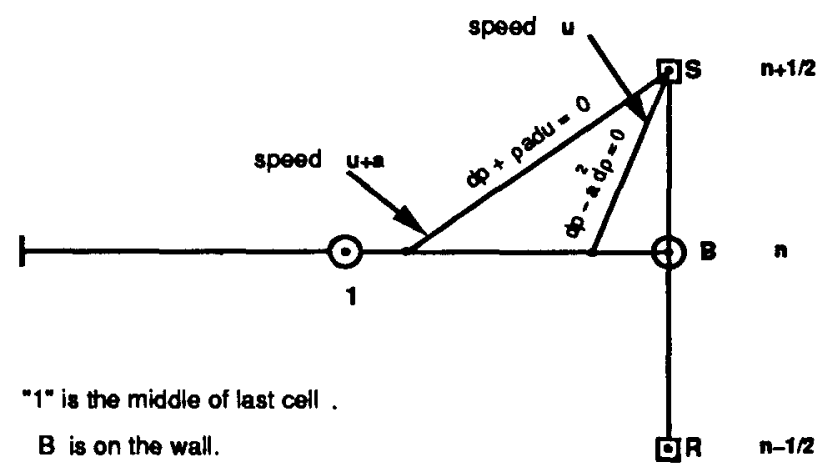

Figure 14: Sketch of the stencil used in boundary procedure discretization

\subsection{Discretization}

Normally, when a cell interface coincides with a solid boundary, it is only necessary to provide some recipe for the pressure on that interface, because all other components of the flux will vanish. For our first-order code, we simply set the wall pressure equal to the pressure in the first cell. Here, we allow that there may be flow through such an interface, and so need to have a complete description of all variables. We would also like to construct the discretisation so that steady solutions are unaffected by the changed boundary condition.

It may be remarked that these inner boundary conditions are easier to discretize than "non-reflecting" outer boundary conditions for two reasons. The first is that waves really do arrive, to a good approximation, normal to the boundary and indeed this approximation improves as we use finer grids. The second is that we have a fine mesh to work on.

The scheme that was used, combined a simple discretisation of eqn 6 with two characteristic equations, one for the incoming acoustic wave (in a onedimensional approximation) and one for the particle path. The stencil used is shown in Fig (14). Point 1 represents the state in the cell next to the wall at the start of the current timestep. Point $R$ represents the boundary flux used in the previous timestep, and $S$ the boundary flux for this time step. Point $B$ is the extrapolation of the current solution to the wall. We will have:

$$
\begin{aligned}
& U_{t}+\frac{U}{\tau}=\frac{\mu}{\rho a} P_{t}(10) \\
& \frac{U_{S}-U_{R}}{\Delta t}+\frac{U_{S}+U_{R}}{2 \tau}=\frac{\mu}{\rho_{R} a_{R}} \frac{P_{S}-P_{R}}{\Delta t} \\
&\left(1+\frac{\Delta t}{2 \tau}\right) U_{S}-\frac{\mu}{\rho_{R} a_{R}} P_{S}=\left(1-\frac{\Delta t}{2 \tau}\right) U_{R}-\frac{\mu}{\rho_{R} a_{R}} P_{R}
\end{aligned}
$$


Here, and elsewhere, we use conditions at $\mathbf{R}$ to evaluate the coefficients of the equation; this makes the resulting system of equations linear. Then we use the following characteristic differential equations, which are respectively correct on $d x / d t=U$ and $d x / d t=U+a$ :

$$
\begin{aligned}
& d P-a^{2} d \rho=0 \\
& \left(P_{t}-a^{2} \rho_{t}\right)+U\left(P_{x}-a^{2} \rho_{x}\right) \quad=0 \\
& \left(\frac{P_{S}-P_{R}}{\Delta t}-a_{R}^{2} \frac{\rho_{S}-\rho_{R}}{\Delta t}\right) \\
& +U_{R}\left(\frac{P_{B}-P_{1}}{\Delta x / 2}-a_{R}^{2} \frac{\rho_{B}-\rho_{1}}{\Delta x / 2}\right)=0 \\
& d P+\rho a d U=0 \\
& \left(P_{t}+\rho a U_{t}\right)+(U+a)\left(P_{x}+\rho a U_{x}\right) \\
& \left(\frac{P_{S}-P_{R}}{\Delta t}+\rho_{R} a_{R} \frac{U_{S}-U_{R}}{\Delta t}\right) \\
& +\left(U_{R}+a_{R}\right)\left(\frac{P_{B}-P_{1}}{\Delta x / 2}+\rho_{R} a_{R} \frac{U_{B}-U_{1}}{\Delta x / 2}\right)=0
\end{aligned}
$$

Now remove values at point $B$ by interpolating between $S$ and $R$, i.e. substitute $U_{B}=\frac{U_{S}+U_{R}}{2}$. Introduce the variables:

$$
\begin{aligned}
\nu_{1} & =\frac{\Delta t U_{R}}{\Delta x} \\
\nu_{2} & =\frac{\Delta t}{\Delta x}\left(U_{R}+a_{R}\right) \\
\mu^{\prime} & =\frac{\mu}{\rho_{R} a_{R}}
\end{aligned}
$$

The equations may then be rearranged as

$$
\begin{gathered}
(\tau+\Delta t / 2) U_{S}-\tau \mu^{\prime} P_{S}=(\tau-\Delta t / 2) U_{R}-\tau \mu^{\prime} P_{R} \\
\left(1+\nu_{1}\right)\left[P_{S}-a_{R}^{2} \rho_{S}\right]= \\
\left(1-\nu_{1}\right)\left[P_{R}-a_{R}^{2} \rho_{R}\right]+2 \nu_{1}\left[P_{1}-a_{R}^{2} \rho_{1}\right] \\
\left(1+\nu_{2}\right)\left[P_{S}+\rho_{R} a_{R} U_{S}\right]= \\
\left(1-\nu_{2}\right)\left[P_{R}+\rho_{R} a_{R} U_{R}\right]+2 \nu_{2}\left[P_{1}+\rho_{R} a_{R} U_{1}\right]
\end{gathered}
$$

and solved directly to find conditions at $S$. At convergence, when conditions at $S$ and $R$ are the same, we see from these equations that

$$
\begin{aligned}
U_{S} & =0 \\
P_{S}-a_{R}^{2} \rho_{S} & =P_{1}-a_{R}^{2} \rho_{1} \\
P_{S}+\rho_{R} a_{R} U_{S} & =P_{1}+\rho_{R} a_{R} U_{1}
\end{aligned}
$$

Although the last of these might be a resonable boundary condition (to first order) it does not coincide with the condition $P_{S}=P_{1}$ used in our regular code. Therefore we replaced $U_{1}$ by $U_{R}$ in the last term of eq 14 . This created no stability problems, and we were able to obtain identical converged solutions from both the soft-wall and solid-wall version of the code.

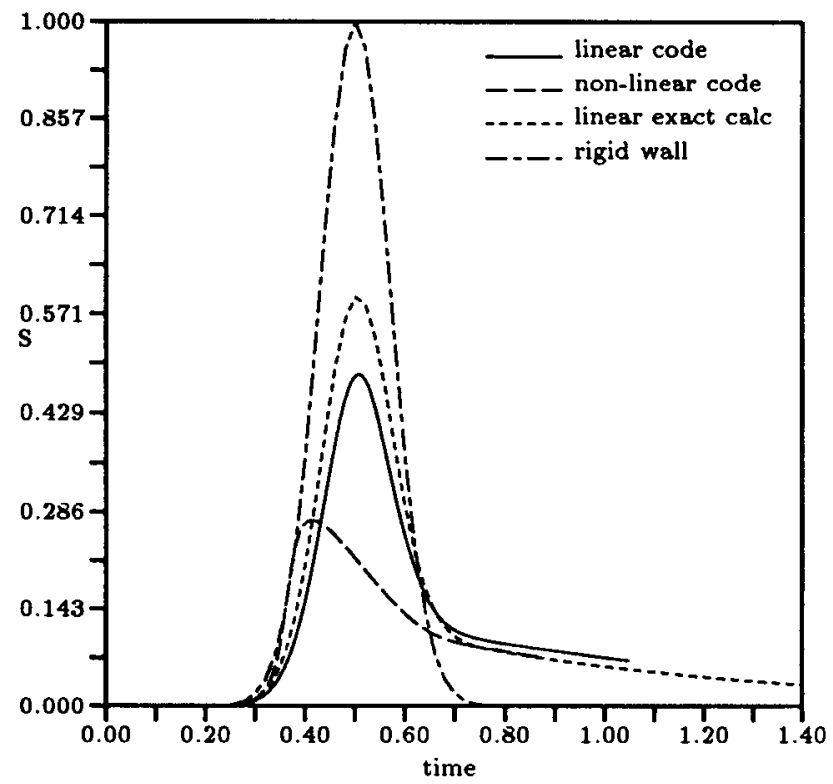

Figure 15: Comparison of $S(t)$ for Gaussian initial condition with $\bar{p} / p_{0}=0.8$

\subsection{Numerical Experiments}

To check how this works at the discrete level, two sets of experiments were accomplished. In the first experiment, the suggested boundary conditions were used for the one-dimensional linear Euler equations (equation (7)) in the domain $x<0, t>0$. We used characteristic equations for the remaining required boundary condition, and applied conventional discretizations. As expected, for all different values of $\tau$ and $\mu$, results in complete consistency with the above exact analytical calculations were obtained (i.e. the reflected amplitude and also, the optimal values for $\tau$ and $\mu$ agree with the theory ).

The second set of experiments was to solve the full Euler equations in the same domain as the previous experiment. This time, having required one more boundary condition, we used two characteristic equations to prepare the complete set of required boundary equations. These experiments were to test the boundary procedure developed in section 3.2.

Many experiments were executed for low and high amplitude initial pressure distribution. For the low amplitude waves, for nearly all values of $\tau$ and $\mu$ behaviour similar to the exact solution was observed. For high amplitude initial waves, as expected, qualitative agreement was seen (see Figs 15, 16,17).

Fig 15 compares $S(t)$ for Gaussian initial condition for a high amplitude wave $\left(\vec{p} / p_{0}=0.8\right)$ obtained by the 


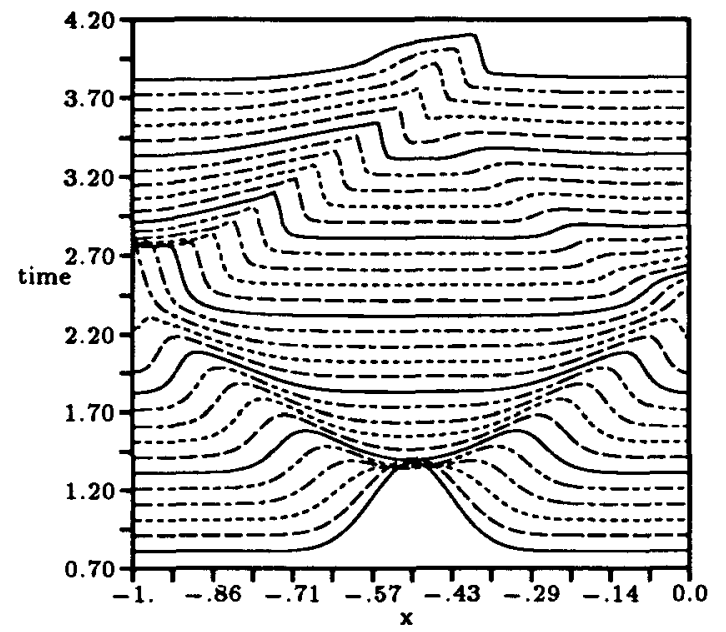

Figure 16: Pressure distribution evolution. The RHS boundary condition is the new BC with $\tau / \Delta t_{\text {cell }}=80$ $\mu=0.3$, and the LHS is a rigid wall. Note: $\bar{p} / p_{0}=0.8$

following ways:

- a) Using new boundary conditions with $\mu=0.3$ $\tau / \Delta t_{c e l l}=80$

1. Numerical experiment for linear equations

2. Numerical experiment for non-linear equations

3. Exact calculations for linear equations

- b) Exact calculations for linear equations for a rigid wall

Since the problem is now strongly non-linear, the theoretical prediction is only qualitative. However, we found that the trends with respect to $\tau$ and $\mu$ were preserved; in particular the optimum values were nearly unaltered.

Figs 16 and 17 will give a more complete picture of the way that the new boundary condition behave. In Fig 16, non-optimal choice of parameters allows partial reflection of waves from the right-hand boundary. In Fig 17 we take the near-optimal choices $\tau / \Delta t_{c e l l}=300$ and $\mu=1.0$, then it is seen that this high amplitude initial disturbance is very strongly damped. Actually, in applications reported below, only weak disturbances need to be treated.

To sum up, these one-dimensional experiences show that a "soft wall" boundary condition can be implemented, and can greatly reduce wave reflections while ultimately achieving the same steady state. In the next section, we will apply these results to two-dimensonal flow.

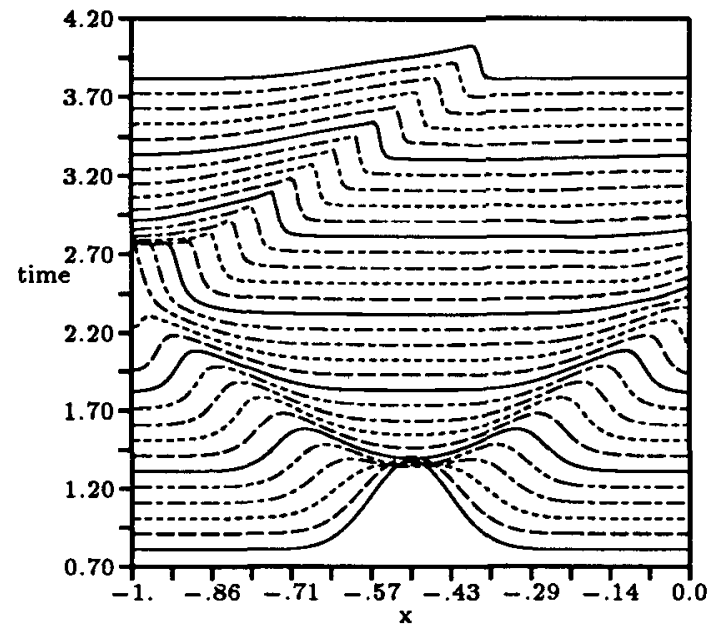

Figure 17: Pressure distribution evolution. The RHS boundary condition is the new BC with $\tau / \Delta t_{c e l l}=300$ $\mu=1.0$, and the LHS is a rigid wall. Note: $\bar{p} / p_{0}=0.8$

\section{Applications}

The testing ground for these ideas in two dimensions was the same airfoil code used to determine the residual histories in section 2.1. The only modification made was to replace the simple wall boundary conditions

$$
\begin{aligned}
\left(u_{n}\right)_{S} & =0 \\
P_{S} & =P_{1}
\end{aligned}
$$

with the eqn 14. A typical result is shown in Fig 19, which is directly comparable with Fig 1 . For this experiment, a $64 \times 32$ O-type grid on a NACA0012 airfoil was used. Also, $M=0.63$ and the angle of attack is 2 degrees. Again, the code is a first order upwind scheme, using Roe's FDS method. The parameters used were $\mu=0.5$ and $\tau / \Delta t_{c e l l}=100$. The value of $\mu$ and $\tau / \Delta t_{c e l l}$ were found experimentally, although with hindsight the arguments in sec 3.1 could have been used to justify the choice of $\mu$. A simple argument to motivate the choice of $\tau / \Delta t_{\text {cell }}$ is as follows.

Under local timestepping, with Courant number $\nu$, the number of iterations required for a wave to pass downstream from the trailing edge to the outer boundary, or from the outer boundary to the leading edge, will be roughly $N_{R} / \nu$, where $N_{R}$ is the number of cells in the radial direction. To travel in the reverse direction will take $\left(N_{R} / \nu\right)[(1+M) /(1-M)]$ iterations, giving a total of $2 N_{R} /[\nu(1-M)]$. To ensure that the boundary perturbation dies away before the next wave returns, we require

$$
\exp \left\{-2 N_{R}\left(\Delta t_{c e l l} / \tau\right) /[\nu(1-M)]\right\} \ll 1
$$




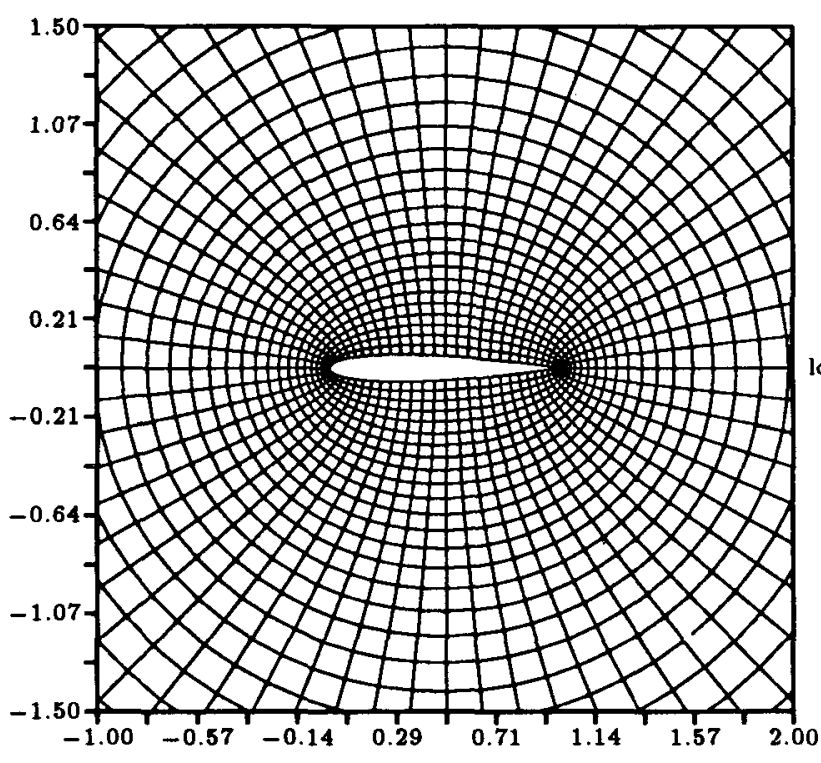

Figure 18: The grid that was used in computation, $64 \times$ $32, R_{\text {out }}=5 \times$ chord

or, say

$$
\frac{2 N_{R} \Delta t_{c e l l}}{\nu(1-M) \tau}>2
$$

whence

$$
\frac{\tau}{\Delta t_{\text {cell }}}<\frac{N_{R}}{\nu(1-M)}
$$

The evolution divides into three phases. In the first phase, no advantage is seen for the soft boundary. In the second phase, begining with the first arrival of returning waves at the airfoil, the residual for the soft boundary is substantially reduced, and the oscillations are much less marked. The video, however, shows that the recurent wave pattern is still present. Fig 20 shows that the lift coefficient is slower to build up with the soft boundary, but converges more steadily. After about 1400 iterations, the residual for the soft boundary has been reduced by about six orders of magnitude, which might be enough to justify halting the code. With the rigid boundary, the same reduction is not achieved untill about 2300 iterations, which is a saving of about $40 \%$. Very similar results are achieved with any change of parameters in the range $0.7>\mu>0.4$ and $300>\frac{T}{\Delta t_{c e l l}}>50$.

After this time, a third phase sets in, during which the soft boundary again shows only slight advantage. This seems to be because its work is essentially over. Video film of this phase shows that the soft-wall calculation no longer features any noticeable wave propagation. Instead, a rather static pattern of residuals appears on the screen, and is rather slowly damped.

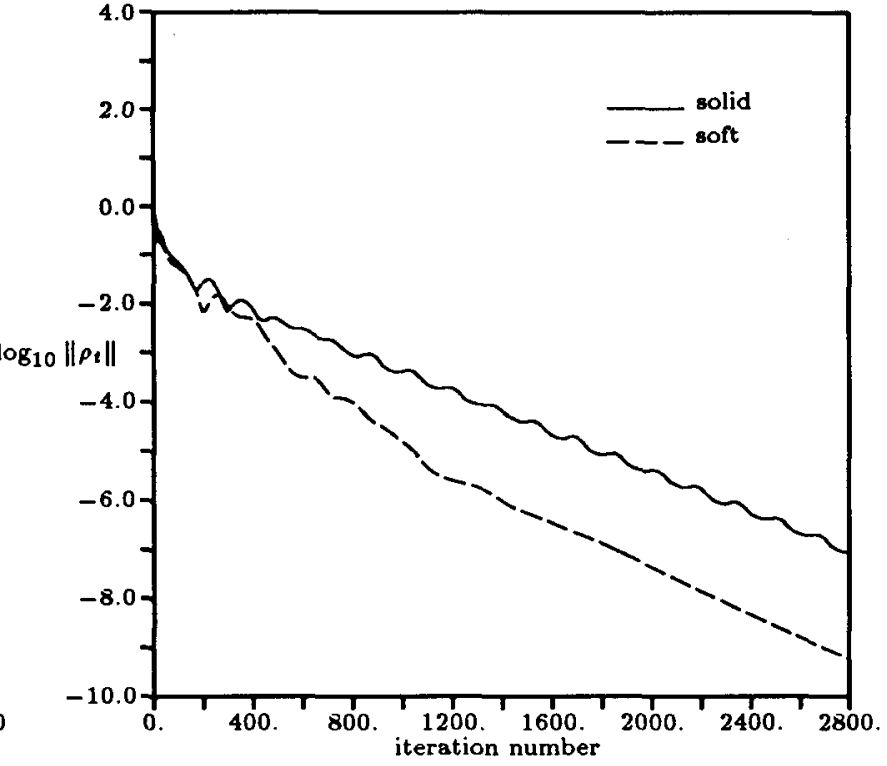

Figure 19: Comparison of Residual history for solid and soft inner boundary condition

The flux through the wall also continues to diminish (Fig 21). This last phase of convergence is not seen in single-precision calculations, because machine accuracy is reached first. At the present time, we can not give any more detailed observations than this.

We have not yet made extensive experiments on finer grids or grids with more remote boundaries. Preliminary results, however indicate that the gains are even greater on such grids, because the waves return at longer intervals, and the wall has time to "set" before they arrive. We have seen gains of $50-60 \%$. However, the pattern of the residual waves becomes more complicated.

\section{Conclusions}

We have presented evidence that convergence of a simple airfoil calculation to the steady state is dominated by the repeated passage of acoustic waves between the inner and outer boundaries. The waves are locked into a single dominant frequency by a wave focusing mechanism that arises from local timestepping. To reduce the waves amplitude, we have designed a "soft wall" boundary condition that reduces wave reflections whilst preserving the steady state solution. One-dimensional linearized analysis helps to select effective boundary parameters. A very simple and inexpensive modification to an existing code saves at least $40 \%$ of the run-time 


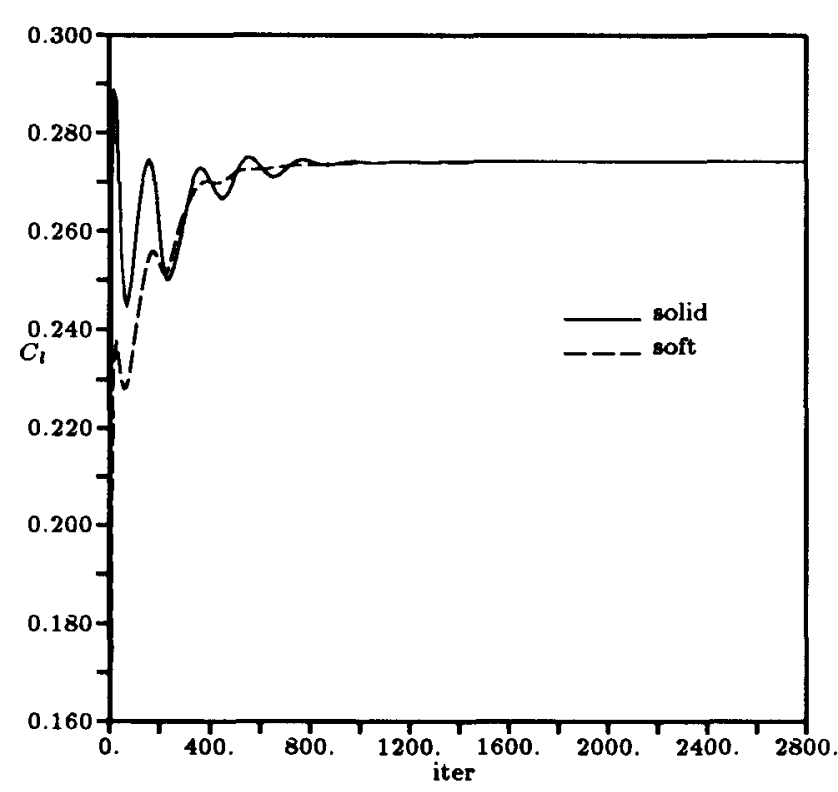

Figure 20: Comparison of $C_{l}$ history for solid and soft inner boundary condition

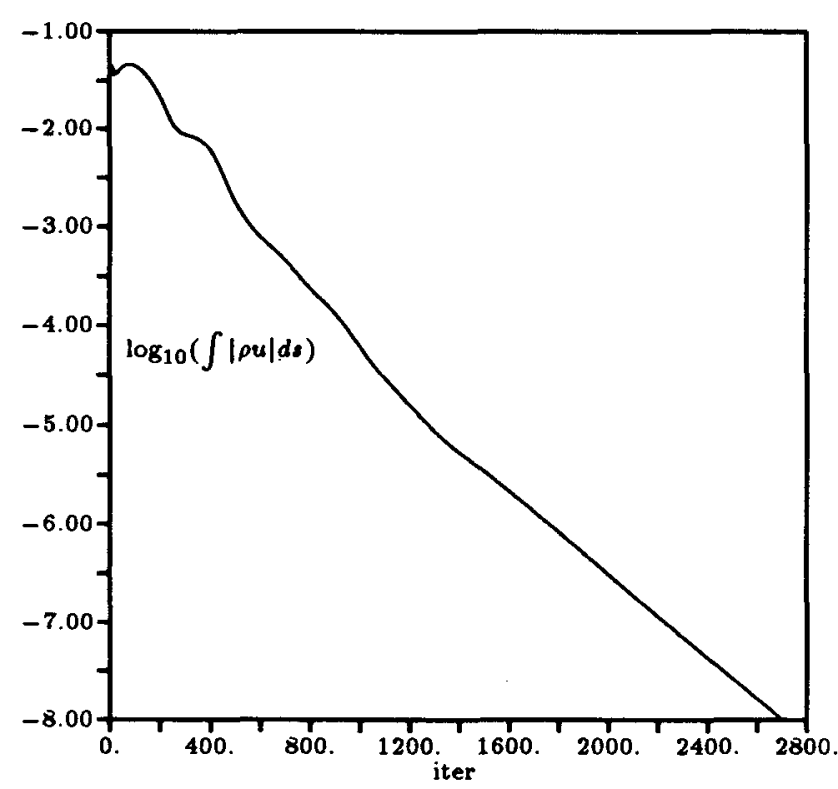

Figure 21: The integral of normal flux on the inner boundary. The integral is scaled by $2 \times \operatorname{chord} \times\left(\rho_{\infty} a_{\infty}\right)$ needed for well-converged solutions. Because the mechanism we exploit is entirely local, we expect that these improvements can be added to, and will not interfere with, other improvements made on completely different lines. These include the modified outer boundary condition of Karni [5] which is a natural complement to this work, and the characteristic time-stepping of van Leer etal [6] which also seems to give rise to sharply tuned residual waves.

\section{Acknowledgements}

The authors are grateful to Professors Kenneth G. Powell and Smadar Karni for helpful discussions.

\section{References}

[1] A. Jameson, W. Schmidt, and E. Turkel, "Numerical solutions of the Euler equations by a finitevolume method using Runge-Kutta time-stepping schemes," AIAA Paper 81-1259, 1981.

[2] A. Bayliss and E. Turkel, "Far-field boundary conditions for compressible flows," Journal of Computational Physics, vol. 48, 1982.

[3] P. L. Roe, "Remote boundary conditions for unsteady multidimensional aerodynamic computations," Computers and Fluids, vol. 17, 1989.

[4] E. Harabetian, "Propagation of singularities, Hamilton-Jacobi equations, and numerical applications," Submitted to Transactions of American Mathematics Society, 1991.

[5] S. Karni, "Accelerated convergence to steady states by gradual far-field damping," in AIAA 10th Computational Fluid Dynamics Conference, 1991.

[6] B. van Leer, W. T. Lee, and P. L. Roe, "Characteristic time-stepping or local preconditioning of the Euler equations," in AIAA 10th Computational Fluid Dynamics Conference, 1991. 